\title{
SUPERFLUIDITY OF HEATED FERMI SYSTEMS IN STATIC FLUCTUATION APPROXIMATION
}

\author{
Khamzin A. ${ }^{1}$, Nikitin A.S. ${ }^{2}$, Sitdikov A.S. ${ }^{2}$ \\ ${ }^{1}$ Kazan (Volga region) Federal University, Institute of Physics, Russia \\ ${ }^{2}$ Kazan State Power-Engineering University, Russia \\ E-mail: airat.khamzin@rambler.ru
}

The strongly excited systems have an extremely high level density and therefore some problems concerning to realistic calculations in the framework of microcanonical ensemble appear in their theoretical studies. Due to this fact, it seems more expedient to use the statistical description. Therefore, building an effective statistical theory, which describes wide range of phenomena in heated Fermi systems, is a very actual problem.

During the investigations of the properties of high excited finite Fermi systems the methods and approximations developed for weakly excited systems (at $T=0$ ) are usually generalized. But many of the assayed approximations does not allow to take correctly into account the correlation effects of different types, which can play an important role at explaining the observable statistical properties.

For investigations of the thermodynamical properties of heated finite Fermi systems we suggest the original method, i.e. the method of statistic fluctuation approximation (SFA). This method is based on the replacement of the square of the deviation from the mean value of the local field operator by the mean value of that square. In this replacement the exact quantum mechanical spectrum of the local field is replaced by its distribution, for which we can count the moments of second order. As a result it becomes possible to consider the fluctuations of local field. As it is shown by calculations of thermodynamical characteristics of different many-body systems with strong interactions, it becomes sufficient for correct consideration of correlation effects.

For Fermi systems, which is in a bound state due to the two-particle interactions, in a framework SFA we obtain closed self-consistent system of equations for calculating the correlation functions, average energy, chemical potential, entropy and other characteristics as temperature functions with including paring between particles. Also the influence of the fluctuations to temperature behavior of the energy gap and the thermal capacity is demonstrated. 